\title{
Efficient Non-Local Image Denoising Using Binary Descriptor Classification
}

\author{
Jan-Ray Liao ${ }^{\mathrm{a}, \mathrm{b}, *}$, Ya-Wei Tsai ${ }^{\mathrm{b}}$, Yu-Hsin Huang ${ }^{\mathrm{b}}$ \\ anstitute of Communication Engineering, National Chung Hsing University, Taichung 402, Taiwan \\ bDepartment of Electrical Engineering, National Chung Hsing University, Taichung 402, Taiwan \\ *Corresponding Author: jrliao@mail.nchu.edu.tw
}

\begin{abstract}
Non-local mean (NLM) is one of the most effective image denoising methods currently available. It calculates weights of neighboring pixels based on the similarity between two image patches. The pixel is then estimated by the weighted sum of the neighboring pixels and itself. Because the large number of the patches needs to be compared, NLM incurs extremely high computational cost. In this paper, we propose to use binary descriptors to reject dissimilar patches from the computation and improve the performance of NLM. A binary descriptor of a patch compares the pixels in the patch with a given threshold and produces a simple binary string to describe the patch. It is very simple to generate and the comparison between two descriptors is computationally efficient. Experimental results show that using a simple binary descriptor can effectively increase the denoising performance of NLM and significantly reduce the execution time.
\end{abstract}

Keywords: Denoising, Non-Local Mean, Binary Descriptor, Pre-classification.

\section{Introduction}

With the popularity of digital imaging devices, image denoising has become an important application in image processing. One of the most effective image denoising methods is the non-local mean (NLM) ${ }^{(1,2)}$. For a pixel to be denoised, NLM first calculates weights for its neighboring pixels based on the similarity between the image patch of the current pixel and the image patches of the neighboring pixels. The current pixel is then replaced by the weighted sum of its neighboring pixels and itself. Because pixels from similar patches are given large weights, the collection of similar pixels can effectively remove unwanted noise in practice.
Because the area of the neighborhood to be searched usually needs to be sufficiently large so that the non-local nature of the algorithm can be exploited, a large number of image patches need to be compared and their corresponding weights to be calculated in order to find the weighted sum of the current pixel. This means that NLM incurs extremely high computational cost and leads to many researches in reducing the computational burden. These acceleration methods can be divided into three categories. The first category is to avoid dissimilar patches by pre-classification ${ }^{3-}$ 9). The criteria used to reject image patches include: means $^{(3,5,7)}$, variances ${ }^{(5,7)}$, higher-order statistical moments $^{(4,6)}$, gradients $^{(3)}$, and pixel intensities ${ }^{(8,9)}$. The second category is to reduce the dimensions of the image patches so that the comparison between patches can be simplified $^{(10-15)}$. The dimension-reduction techniques include principle component analysis ${ }^{(10,11,13-15)}$ and singular value decomposition ${ }^{(12)}$. The third category is to speed up the computation by using either efficient algorithms or data structures $^{(16-23)}$. The efficient algorithms being used are random selections ${ }^{(16,23)}$, fast Fourier transform ${ }^{(17,18,22)}$, squared image ${ }^{(18,19)}$, and probabilistic early termination ${ }^{(21)}$. The data structures for improving efficiency include Laplacian pyramid ${ }^{(18)}$ and multiresolution representations ${ }^{(20)}$.

Among these three categories of acceleration techniques, the first category has an additional advantage of producing images with less blurring. This is because that the small weights given to pixels from dissimilar patches eventually accumulate to a significant amount and bias the estimation ${ }^{(24)}$. By rejecting dissimilar patches in the weight-calculating process, the bias from these small weights can be removed and result in an image of better quality. However, in order for the pre-classification to work, additional statistical parameters such as means or gradients need to be calculated before the NLM process. This adds additional complexity in the algorithm. Therefore, the motivation of this paper is to 
find a simplified parameter for the pre-classification process while still maintain the advantages of more complex parameters. To this end, we propose to use binary descriptors in the pre-classification of NLM. A binary descriptor of an image patch compares the pixels in the patch with a given threshold and produces a simple binary string to describe the patch. The reasons to choose binary descriptors as an alternative to other statistical parameters are that they are very simple to generate and the cost of comparison between two descriptors is extremely low.

In this paper, we study two kinds of binary descriptor. The first is called "simple binary pattern" (SBP) which uses half of the maximum pixel value as the threshold. The second is a very popular binary descriptor called "local binary pattern" (LBP) ${ }^{(25)}$ which uses the value of center pixel as the threshold. Only image patches with the same description are considered in the weight calculation. The experimental results show that using SBP can effectively increase the denoising performance of NLM and significantly reduce the execution time.

The rest of the paper is organized as follows. In the second section, we present the binary descriptors and the modified NLM algorithm used in this paper. In the third section, we show the experimental results. In the fourth section, we conclude.

\section{Non-Local Mean and Binary Descriptors}

\subsection{Non-Local Mean}

Let $X$ be the original noise-free image, $\hat{X}$ be the estimated image from a noisy image $Y$ which is interfered by an additive Gaussian noise with zero mean and variance $\sigma^{2}$. We assume that the pixel values of all the images are normalized to the range between 0 and 1 . Let $\mathbf{m}=(x, y)$ and $\mathbf{n}=\left(x^{\prime}, y^{\prime}\right)$ denote two locations in the images. Let $Y(\mathbf{m})$ denote the pixel value at location $\mathbf{m}$ and $\mathbf{Y}_{\mathbf{n}}$ denote the image patch which is centered at location $\mathbf{n}$. The patch $\mathbf{Y}_{\mathbf{n}}$ extends to $k$ pixels around $\mathbf{n}$ in all four directions (top, bottom, left, and right), i.e., the size of the patch is $(2 k+1)^{2}$.

The NLM algorithm works as follows:

For a location $\mathbf{m}$, let $S_{\mathbf{m}}$ be a square search window centered at $\mathbf{m}$ and extended to $s$ pixels in all four directions, i.e., the size of the search window is $(2 s+1)^{2}$. For each patch $\mathbf{Y}_{\mathbf{n}}$ where $\mathbf{n} \in S_{\mathbf{m}}$, the weight is calculated as:

$$
w_{\mathbf{n m}}=\exp \left(-\frac{\left\|\mathbf{Y}_{\mathbf{n}}-\mathbf{Y}_{\mathbf{m}}\right\|^{2}}{h^{2}}\right)
$$

where $\|\bullet\|^{2}$ is the Euclidean norm and $h$ is an adjustable parameter.

Then, the estimated pixel value is the weighted mean:

$$
\hat{X}(\mathbf{m})=\frac{\sum_{\mathbf{n} \in S_{\mathbf{m}}} w_{\mathbf{n m}} Y(\mathbf{n})}{\sum_{\mathbf{n} \in S_{\mathbf{m}}} w_{\mathbf{n m}}}
$$

\subsection{Binary Descriptors}

A binary descriptor of an image patch compares the pixels within the patch with a given threshold and produces an integer to describe the patch.

Let $q(\bullet)$ be a binary operator (or a quantizer) defined as below:

$$
q(Y(\mathbf{n}))=\left\{\begin{array}{ll}
1, & Y(\mathbf{n}) \geq T_{h} \\
0, & Y(\mathbf{n})<T_{h}
\end{array} .\right.
$$

The relative positions of the pixels to be considered in the description with respect to the center of the patch are given by a set of vectors $V$ :

$$
V=\left\{\mathbf{v}_{i}=\left(d x_{i}, d y_{i}\right), i=1, \cdots, K\right\},
$$

For example, if the center of patch and its four neighbors are considered in the description, the set $V$ is:

$$
V=\{(0,0),(-1,0),(1,0),(0,-1),(0,1)\} .
$$

In this paper, we considered four variations of neighbors in the description. We called these variations: 4-neighbor, 8neighbor, 12-neighbor, and 16-neighbor descriptions. The pixels used in these four variations are shown in Fig. 1.

The description for a patch $\mathbf{Y}_{\mathbf{m}}$ is then calculated as:

$$
D\left(\mathbf{Y}_{\mathbf{m}}\right)=\sum_{i=1}^{K} q\left(Y\left(\mathbf{m}+\mathbf{v}_{i}\right)\right) \text {. }
$$

To put it simply, the description calculates the number of ones within the pixels being considered. Some descriptors multiply a weight of $2^{i-1}$ to each of the quantized values in the summation of (4). However, adding the weights leads to 


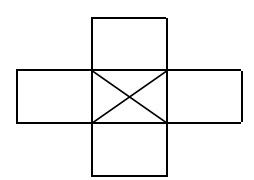

(a)

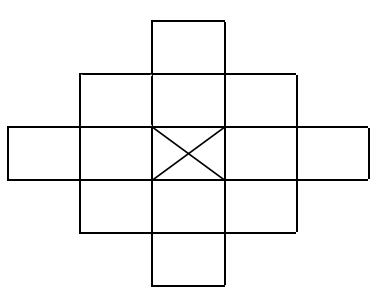

(c)

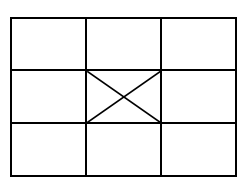

(b)

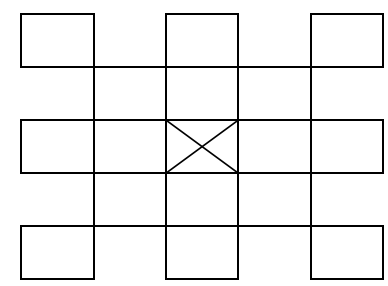

(d)
Fig. 1. The relative positions of the pixels consider in the description. The cross indicates the location of the center pixel: (a) 4-neighbor, (b) 8-neighbor, (c) 12-neighbor, and (d) 16-neighbor descriptions.

too many different classes in the description and, in turn, results in too few patch matches in NLM. We find that adding weights in the calculation does not produce desirable results in denoising. Therefore, the weights are not included in the description.

Two kinds of binary descriptor are considered in this paper. The first is called "simple binary pattern" (SBP) and its threshold, $T_{h}$, in (3) is equal to 0.5 . The second is called "local binary pattern" (LBP) and its threshold is equal to the value of the center pixel, $Y(\mathbf{m})$.

\subsection{Non-Local Mean Algorithm with Binary Descriptor Classification}

With the binary descriptors defined, the original NLM algorithm is modified as follows:

Only when $D\left(\mathbf{Y}_{\mathbf{m}}\right)$ is equal to $D\left(\mathbf{Y}_{\mathbf{n}}\right)$, the weight $w_{\mathbf{n m}}$ in (1) is calculated. Otherwise, the weight $w_{\mathbf{n m}}$ is set to zero. In other words, only patches with the same binary description are included in the denoising calculation of (2).

\section{Experimental Results}

Our proposed algorithm was compared with the original NLM algorithm ${ }^{(1,2)}$ and the accelerated NLM algorithm using the mean of the patch for classification ${ }^{(3,5,7)}$. For all algorithms, $h$ is set to $100 \sigma^{2}$. The mean of a patch is calculated over a 7x7 neighborhood and weights are only calculated for patches with means within $\pm 10 \%$ of the mean of the current patch. These parameters are directly adopted from reference 3 . The reason that mean-classified NLM is used for comparison is that the binary descriptor we used can be viewed as an approximate estimator of the sum of the pixels being considered and this sum is directly related to the mean.

Images from USC-SIPI image database were tested. All algorithms are implemented in $\mathrm{C}$ under Microsoft Visual Studio Community 2015. The test platform is a personal computer with Intel Core i7-4790 CPU running at $3.6 \mathrm{GHz}$ and 8 GB of memory.

Fig. 2 shows the average execution time for all the methods. In the figure, we can see that the execution time for 12 -neighbor SBP is only $28 \%$ of the original NLM and $45 \%$ of the mean-classified NLM. This indicates that SBP NLM does indeed have significant advantage in terms of reducing computational complexity.

Fig. 3 shows PSNR vs. the standard deviation $\sigma$ of the additive Gaussian noise for four of the images tested. From the figure, we can see that 12-neighbor SBP generally performs better than mean-classified NLM when $\sigma$ is less than 40. Even when it is not better than mean-classified NLM when $\sigma$ is larger, it is still better than the original NLM. The largest gap between 12-neighbor SBP NLM and the original NLM is $2.5 \mathrm{~dB}$ which occurs at $\sigma=30$ for the image House. The only exception is for the image Lena but the performance of SBP NLM is still on the par with the original NLM. In the worst case, PSNR of 12-neighbor SBP NLM is $0.57 \mathrm{~dB}$ lower than that of the original NLM which happens at $\sigma=50$ for the image Lena. However, it is worth noting that the execution time for SBP NLM is much less than that of the original NLM as shown in Fig. 2. Therefore, we believe that 12-neighbor SBP NLM is still better than the original NLM even in the worst case scenario. The reason why SBP

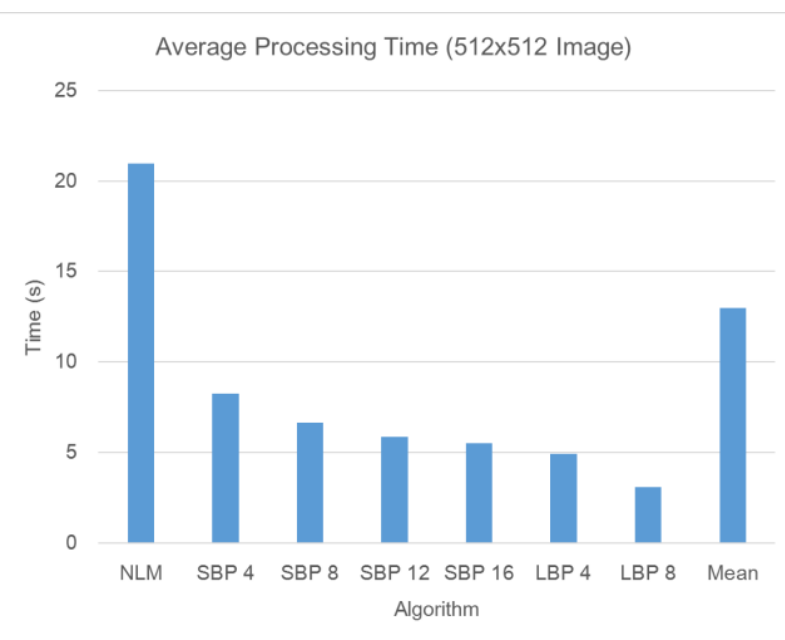

Fig. 2. The average execution time for all the methods 


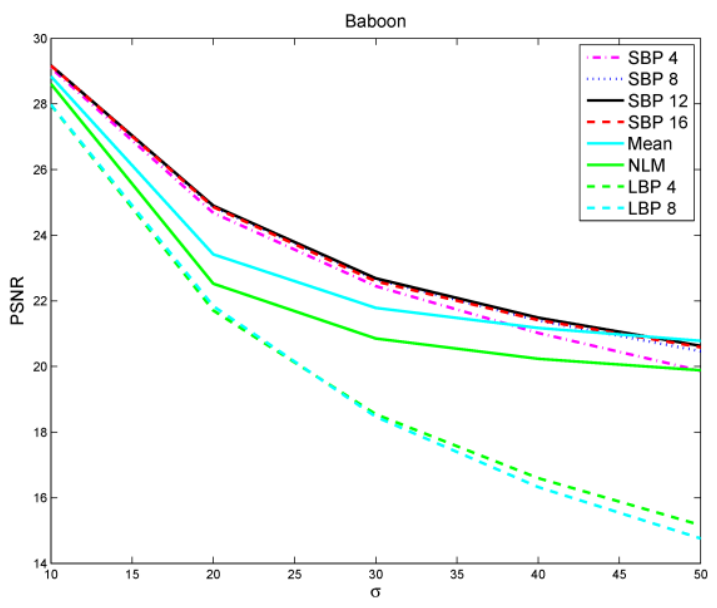

(a)

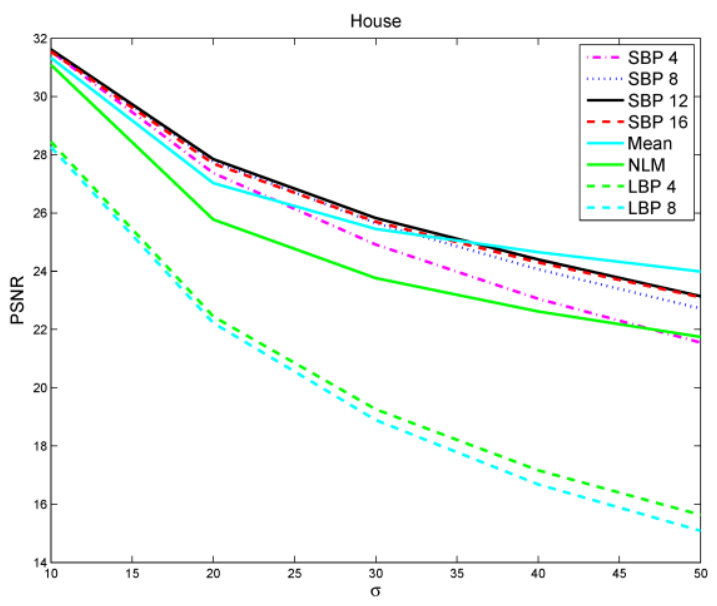

(c)

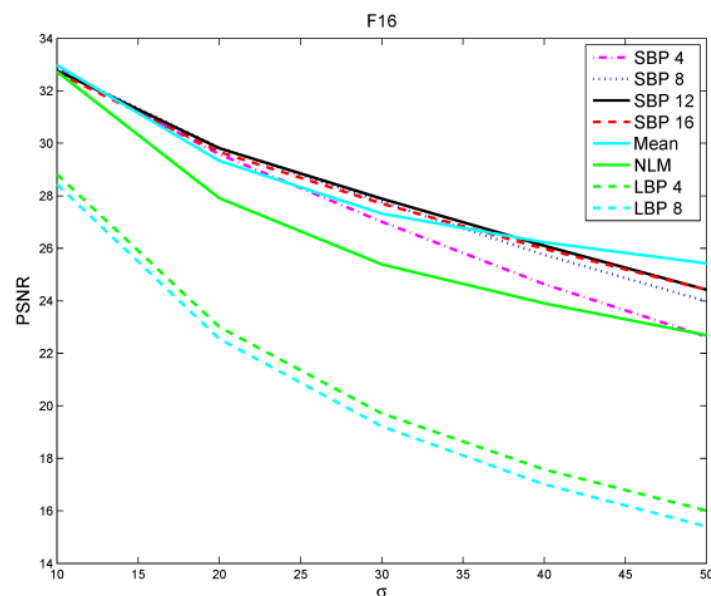

(b)

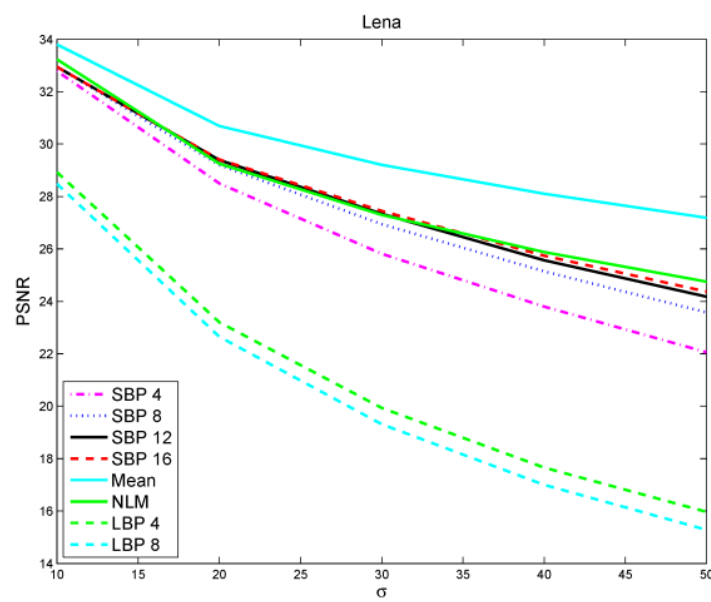

(d)

Fig. 3. PSNR vs. standard deviation of additive Gaussian noise for four images:.(a) Baboon, (b) F-16, (c) House, and (d) Lena.

NLM does not perform as well as mean-classified NLM in some images still needs further investigation. On the other hand, the performance of LBP NLM is worse than the other approaches. Therefore, we conclude that LBP is not suitable as a classifier in NLM.

Fig. 4 shows the images produced by the original NLM, the mean-classified NLM, the 12-neighbor SBP NLM, and the 4-neighbor LBP NLM when $\sigma=30$. From the figures, we can see that SBP can preserve the details of the image much better than the other approaches. This fact is the most distinctive in the image Baboon. Therefore, despite the fact that PSNR for SBP is not better than the original NLM for the image Lena. Subjectively, SBP is still competitive.

The reason that SBP is effective can be attribute to the fact that SBP can be viewed as a mean estimator for the quantized values of the pixels. In other words, SBP provides an approximation of the original mean. Therefore, it can approach the performance of mean-classified NLM when the approximation is accurate. On the other hand, the purpose of an LBP is to describe the relationship between pixels and it appears that this relationship is not a good classifier for NLM. Therefore, our conclusion is that SBP is a good classifier in accelerating NLM.

\section{Conclusions}

In this paper, we show that using a simple binary descriptor to reject dissimilar patches from consideration can significantly reduce the computation time while still increase the denoising performance in NLM. Because binary descriptors are much simpler to calculate than previously proposed parameters such as mean and gradients, it has significant advantage as compared to other approaches in complexity reduction for NLM. 


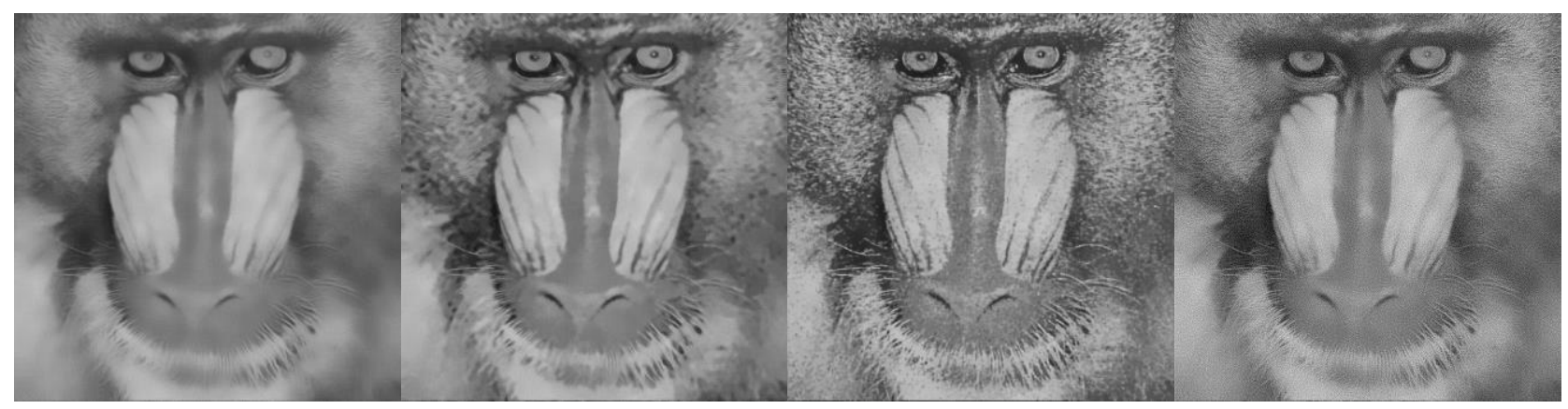

(a)

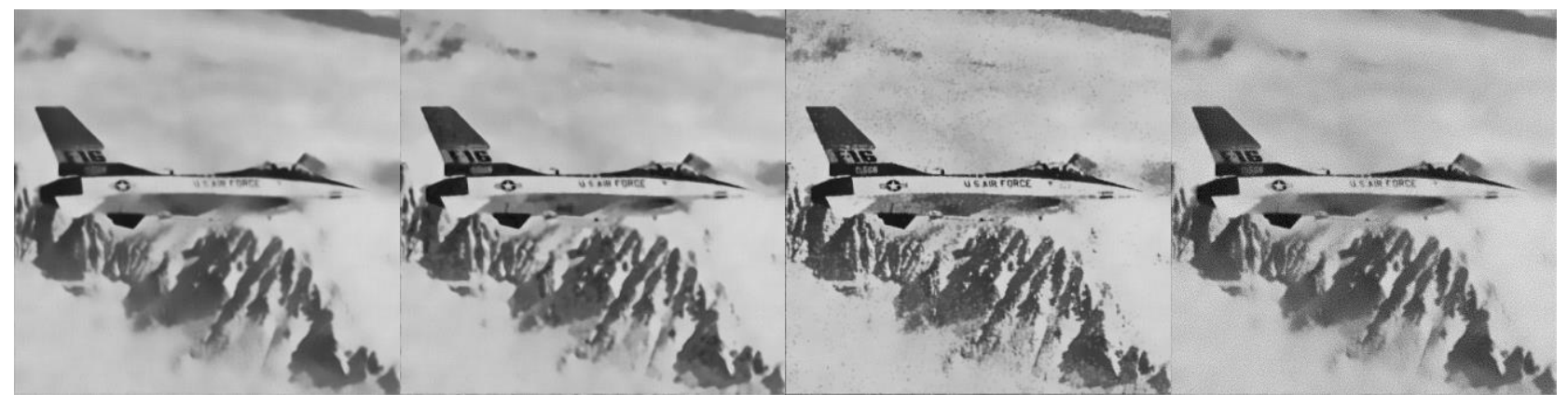

(b)

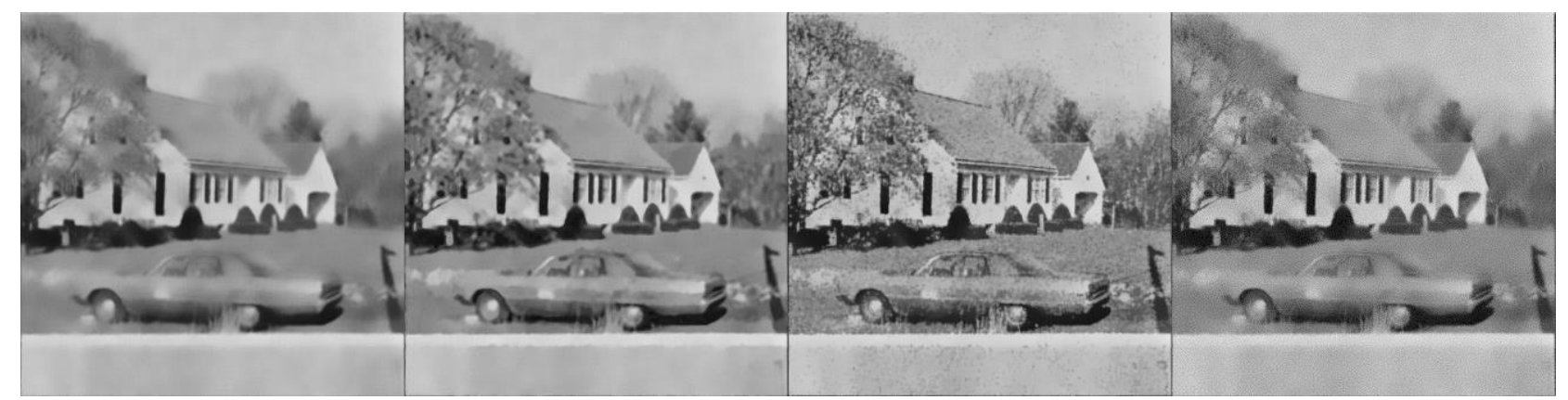

(c)
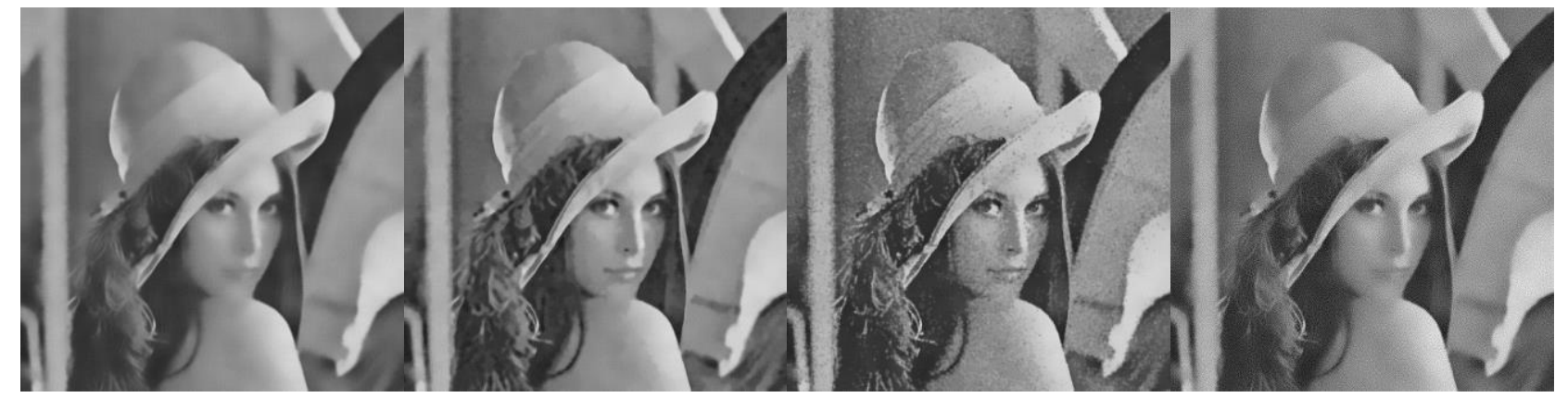

(d)

Fig. 4. Images generated by different methods. From left to right, the methods used are the original NLM, the meanclassified NLM, 12-neighbor SBP NLM, and 4-neighbor LBP NLM:

(a) Baboon, (b) F-16, (c) House, and (d) Lena.

\section{Acknowledgment}

This work was supported by Ministry of Science and Technology, Taiwan, Republic of China, under contract MOST 104-2221-E-005-052.

\section{References}

(1) A. Buades, B. Coll, and J. M. Morel, "A Review of Image Denoising Methods, with a New One," Multiscale Modeling and Simulation, Vol. 4, No. 2, pp. 
490-530, 2005.

(2) A. Buades, B. Coll, and J. M. Morel, "A Non-Local Algorithm for Image Denoising," in Proc. IEEE Computer Vision and Pattern Recognition (CVPR), Vol. 2, Vol. 4191, pp. 60-65, 2005.

(3) M. Mahmoudi and G. Sapiro, "Fast Image and Video Denoising via Nonlocal Means of Similar Neighborhoods," IEEE Signal Processing Letters, Vol. 12, No. 12, pp. 839-842, 2005.

(4) P. Coupe, P. Yger, and C. Barillot, "Fast Non Local Means Denoising for 3D MR Images," in Proc. Medical Image Computing and Computer-Assisted Intervention (MICCAI), Vol. 4191, pp. 33-40, 2006.

(5) C. Kervrann, J. Boulanger, and P. Coupe, "Bayesian Non-Local Means Filter, Image Redundancy and Adaptive Dictionaries for Noise Removal," Proc. 1st Int. Conf. Scale Space and Variational Methods in Computer Vision (SSVM), Vol. 4485, pp. 520-532, 2007.

(6) A. Dauwe, B. Goossens, H. Luong, and W. Philips, "A Fast Non-Local Image Denoising Algorithm," Proc. SPIE, Vol. 6812, pp. 1331-1334, 2008.

(7) T. Brox, O. Kleinschmidt, and D. Cremers, "Efficient Nonlocal Means for Denoising of Textural Patterns," IEEE Trans. Image Processing, Vol. 17, No. 7, pp. 10831092, 2008.

(8) R. C. Bilcu and M. Vehvilainen, "Combined Non-Local Averaging and Intersection of Confidence Intervals for Image De-noising," Proc. IEEE Int. Conf. Image Processing, pp. 1736-1739, 2008.

(9) C. Pang, O. C. Au, J. Dai, Y. Wen, and Z. Feng, “A Fast NL-Means Method in Image Denoising Based on the Similarity of Spatially Sampled Pixels," Proc. IEEE Int. Workshop Multimedia Signal Processing, pp. 1-4, 2009.

(10) N. Azzabou, N. Paragios, and F. Guichard, "Image Denoising Based on Adapted Dictionary Computation," in Proc. IEEE Int. Conf. Image Processesing (ICIP), Vol. 3, pp. 109-112, 2007.

(11) T. Tasdizen, "Principal Components for Non-Local Means Image Denoising," Proc. Int. Conf. Image Processing, pp. 1728-1731, 2008.

(12) J. Orchard, M. Ebrahimi, and A. Wong, "Efficient Nonlocal-Means Denoising Using the SVD," Proc. IEEE Int. Conf. Image Processing, pp. 1732-1735, 2008.

(13) T. Tasdizen, "Principal Neighborhood Dictionaries for Nonlocal Means Image Denoising,"' IEEE Trans. Image Processing, Vol. 18, No. 12, pp. 2649-2660, 2009.

(14) T. Thaipanich, B. T. Oh, P.-H. Wu, D. Xu, C.-C. J. Kuo, "Improved Image Denoising with Adaptive Nonlocal Means (ANLmeans) Algorithm," IEEE Trans. Consumer Electronics, Vol. 56, No. 4, pp. 2623-2630,
2010.

(15)D. Van De Ville and M. Kocher, "Nonlocal Means with Dimensionality Reduction and SURE-Based Parameter Selection," IEEE Trans. Image Processing, Vol. 20, No. 9, pp. 2683-2690, 2011.

(16) N. Azzabou, N. Paragios, F. Guichard, "Random Walks, Constrained Multiple Hypothesis Testing and Image Enhancement," Proc. European Conference on Computer Vision (ECCV), Vol. 1, pp. 379-390, 2006.

(17) J. Wang, Y. Guo, Y. Ying, Y. Liu, and Q. Peng, "Fast Non-Local Algorithm for Image Denoising," Proc. IEEE Int. Conf. Image Processing, pp. 1429-1432, 2006.

(18) Y. L. Liu, J. Wang, X. Chen, Y. W. Guo, and Q. S. Peng, "A Robust and Fast Non-Local Means Algorithm for Image Denoising," Journal of Computer Science and Technology, Vol. 23, No. 2, pp. 270-279, 2008.

(19) J. Darbon, A. Cunha, T. Chan, S. Osher, and G. Jensen, "Fast Nonlocal Filtering Applied to Electron Cryomicroscopy," Proc. 5th IEEE Int. Symp. Biomedical Imaging (ISBI), pp. 1331-1334, 2008.

(20) V. Karnati, M. Uliyar, and S. Dey, "Fast Non-Local Algorithm for Image Denoising," Proc. IEEE Int. Conf. Image Processing, pp. 3873-3876, 2009.

(21)R. Vignesh, B. T. Oh, and C.-C. Kuo, "Fast Non-Local Means (NLM) Computation with Probabilistic Early Termination," IEEE Signal Process. Letters, Vol. 17, No. 3, pp. 277-280, 2010.

(22)C. A. Deledalle, V. Duval, and J. Salmon, "Non-Local Methods with Shape-Adaptive Patches," Journal of Mathematical Imaging and Vision, Vol. 43, No. 2, pp. 103-120, 2012.

(23) S. H. Chan, T. Zickler, and Y. M. Lu, "Monte Carlo Non Local Means: Random Sampling for Large-Scale Image Filtering," IEEE Trans. Image Processing, Vol. 23, No. 8, pp. 3711-3725, 2014. J. Salmon, "On Two Parameters for Denoising with Non-Local Means," IEEE Signal Processing Letters, Vol. 17, No. 3, pp. 269-272, 2010.

(24)T. Ojala, M. Pietikainen, and T. Maenpaa, "Multiresolution Gray-Scale and Rotation Invariant Texture Classification with Local Binary Patterns," IEEE Trans. Pattern Analysis and Machine Intelligence, Vol. 24, No. 7, pp. 971-987, 2002. 